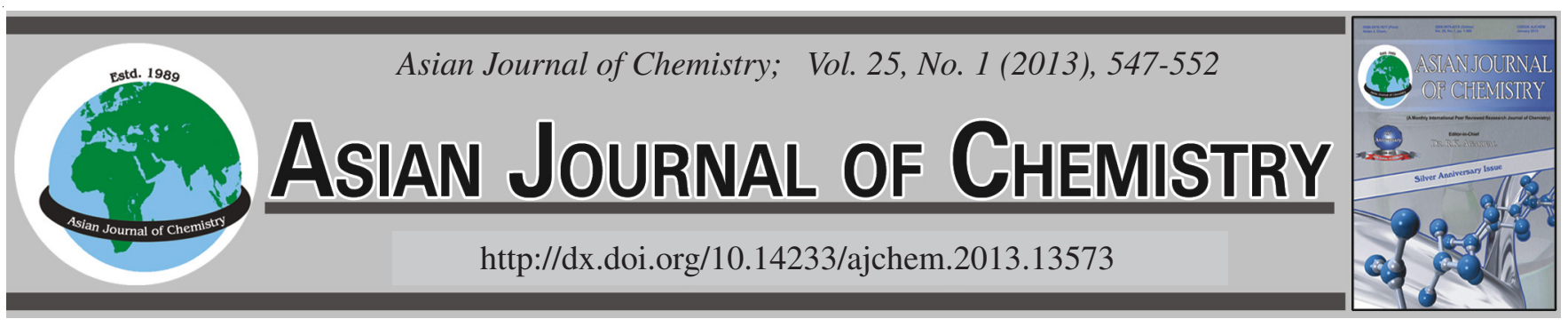

\title{
Induced Orientation in Recrystallization of Polyacrylonitrile Precursor for Carbon Fiber in Steady-State High Magnetic Field
}

\author{
Ren Xiao-Meng*, Wang Yuan-Sheng, Mei Yong-Jia, Xia Zheng-Cai and He Te
}

Naval University of Engineering, Hubei 430033, Wuhan, P.R. China

*Corresponding author: Fax: +86 28 85466331; Tel: +86 187 2850700; E-mail: rxiaomeng@yahoo.cn

\begin{abstract}
Orientation of polyacrylonitrile precursor for carbon fiber was a critical factor to determine the performance of subsequent carbon fiber. In the study, polyacrylonitrile precursor was processed in a steady-state high magnetic field generated by PPMS. The magnetic field intensity was set in $8 \mathrm{~T}, 12 \mathrm{~T}, 16 \mathrm{~T}$ and the processing time was set for $10 \mathrm{~min}, 30 \mathrm{~min}, 50 \mathrm{~min}$, respectively. The results showed that polyacrylonitrile precursor could be induced oriented in recrystallization and both crystalline and overall orientation increased when polyacrylonitrile precursor was processed in magnetic field. The mechanism of crystalline orientation was analyzed from magnetostatic energy, Boltzmann factor and moment of force points of view respectively and the three theories happened to coincide with each other. Moreover, polyacrylonitrile precursor was testified to be diamagnetic anisotropy. It was interesting to detect that the amorphous region was more susceptible to high magnetic field than crystallinity. The increase of overall orientation resulted from amorphous region mainly.

The change of orientation brought about the changes of other properties which were measured by density, DSC, SEM.
\end{abstract}

Key Words: Polyacrylonitrile precursor, High magnetic field, Crystalline orientation, Overall orientation.

\section{INTRODUCTION}

Polyacrylonitrile (PAN) precursor is an important raw material to produce carbon fibers. Large numbers of experiments had confirmed that the structure of polyacrylonitrile precursor determined the performance of subsequent carbon fiber to a great extent. The experimental evidences had implied that the copolymer composition could modify the process and mechanism of the pre-oxidation and then the final carbon fibers would inherit the changes happened in the process $^{1}$. The high molecular weight implied less joint points which would transform to defects in sequent treating process. Besides, the narrow molecular weight distribution could make the defects distribute uniformly ${ }^{2-5}$. Based on the weak-link theory, the strength of the weakest point determined the strength of the whole sample. The uniform distribution of defects avoided the production of weak point and increased the strength of fiber. Besides the factors mentioned above, the recent research had showed that the configuration of molecule especially the orientation is another critical factor to determine the property of polyacrylonitrile precursor ${ }^{6}$. And the orientation could transfer to the pre-oxidation and carbonization fiber, furthermore, have influence on the strength of the ultimate carbon fibers. The experimental results had implied that the defects and voids in polyacrylonitrile precursor would decrease with the improvement of orientation. Tension had been testified to be an effective way to increase orientation of polyacrylonitrile precursor, but the effectiveness was limited because too much tension would cause the fiber cracked.

As the magnetic technique developed, the high magnetic field was applied to the process of polymer increasingly. Especially, when the magnetism was found in metal-free materials, the orientation of organic molecule in magnetic field was verified exactly. The molecules containing aromatic ring were thought to possess magnetic property due to the ring current generated by the delocalization rotation of $\pi$ electron. The a-axis of poly(ethylene terephthalate) crystal aligned along the direction of perpendicular to the magnetic field during the induction period of isothermal crystallization from melt. This result was in accord with the observation that discovered during crystallization of two other crystalline polymer, i.e. poly(ethylene-2,6-naphthalate) and isotactic polystyrene ${ }^{7-9}$. The reason of orientation was attributed to the magnetic anisotropy caused by the induced ring current. Not only aromatic polymer could orient in the high magnetic field, but polymer without aromatic ring could be induced by magnetic treatment. The b-axis of crystal of low weight isotactic polypropylene exhibited orientation along the direction of parallel to the magnetic field. As to high weight polypropylene, it could align when N,N'-dicyclohexyl-2,6-naphthalenendicarbonxamide was add to as the nucleating agent ${ }^{10,11}$. 
In the paper, the polyacrylonitrile precursor was processed in a steady-state high magnetic field generated by PPMS during the process of recrystallization. The magnetic field intensity was set in $8 \mathrm{~T}, 12 \mathrm{~T}, 16 \mathrm{~T}$ and the processing time was set for $10 \mathrm{~min}, 30 \mathrm{~min}, 50 \mathrm{~min}$. The effects of magnetic field on crystalline and overall orientation of polyacrylonitrile precursor were studied. And the mechanism of crystalline orientation was analyzed, the reason for overall orientation was detected and the other properties resulted from the change of orientation were measured.

\section{EXPERIMENTAL}

The polyacrylonitrile precursor (copolymer were IA and EMA, supplied by the Jilin Tangu Co., Ltd., China) was wound in self-made frame and pretreated in oil bath in $180^{\circ} \mathrm{C}$ for $12 \mathrm{~h}$ in order to make the segments relaxed enough and relieve internal stress. Then the sample was put into a selfmade airtight copper pipe which could be heated and full of silicone oil in order to keep the inside temperature uniform. The closed sample tube was fixed on a specialized sample holder and then put into the magnetic field. The steady-state high magnetic field was generated by Physical Property Measurement System (PPMS) containing superconducting magnet (Model 600, Quantum Design Co., USA). The rising rate of magnetic field was set as $100 \mathrm{Oe} / \mathrm{s}$ and the magnetic field lasted for $10 \mathrm{~min}, 30 \mathrm{~min}, 50 \mathrm{~min}$ respectively when the intensity reached to the set value. Meanwhile, the sample was heated to $130^{\circ} \mathrm{C}$ at the rate of $5^{\circ} \mathrm{C} / \mathrm{min}$ in order to keep the temperature above glass transition temperature $\left(\mathrm{T}_{\mathrm{g}}\right.$, about $\left.103{ }^{\circ} \mathrm{C}\right)$. When the process completed, the magnetic field was removed at the rate of $150 \mathrm{Oe} / \mathrm{s}$ and the temperature fell to $25^{\circ} \mathrm{C}$ at the rate of $5{ }^{\circ} \mathrm{C} / \mathrm{min}$. After that, the sample was taken out of the sample tube and washed by cyclohexane in order to remove the silicone oil. When all the process was accomplished, the sample was placed in refrigerator in order to prevent the segments from movement.

Detection method: The crystalline orientation was measured by XRD azimuth angle scan. X-ray diffractometer: type D/max-2550 PC, Rigaku Co., Japan (included accessory for fiber). The test condition: $\mathrm{CuK} \alpha_{1}(\lambda=0.154056 \mathrm{~nm})$ radiation, X-ray unit operated at $40 \mathrm{KV}$ and $300 \mathrm{~mA}$, angular intervals was $0.02^{\circ}$, residence time was $0.12 \mathrm{~s}$. The degree of crystalline orientation was calculate by eqn. 1 ,

$$
\mathrm{f}_{\mathrm{c}}=\frac{360-\sum \mathrm{H}}{360}
$$

where $\mathrm{H}$ was the integral value of full width at half maximum of diffraction peak.

The overall orientation was indicated by the orientation of cyano-group, which was measured by infrared dichroism. Fourier transform infrared spectrometer: type Nicolet 560, Nicolet Co., USA. The sample was fixed to monolayer in a self-made frame. The test condition: the number of scan times was 32 , scan range was $4000-400 \mathrm{~cm}^{-1}$, step was $4 \mathrm{~cm}^{-1}$. The orientation function and orientation angle were calculated by eqn. 2 ,

$$
\mathrm{f}_{\mathrm{r}}=\frac{\mathrm{R}-1}{\mathrm{R}+2} \times \frac{2}{3 \cos ^{2} \alpha-1}
$$

where $R$ was dichroic ratio which was calculated by $A_{\|} / A_{\perp}$ and $A_{\|}, A_{\perp}$ were the absorbance when the fiber was parallel and perpendicular to the incident light. $\alpha$ was the angle between the transition moment of absorption band and the axes of molecular chain and it was $73^{\circ} . \theta$ was the angle between the axes of fiber and the tension direction.

The density was measured by density gradient column which was composed of $n$-heptane and tetrachloromethane. The fiber was cut into 1-2 mm segments and put into the density gradient column. Then the fiber suspended for $4 \mathrm{~h}$ and remained stationary and the graduation value of position in which fiber suspended was the density of fiber.

The thermal behaviour was measured by DSC. Differential scanning calorimetry: type Philx 204, Netzsch Co., Germany. The test condition: the heating rate was $10 \% \mathrm{~min}$ in nitrogen atmosphere, the range of temperature was $30-400{ }^{\circ} \mathrm{C}$, sample was weighed $4.0 \mathrm{mg}$.

The fiber was wrapped by epoxy resin and fractured in liquid nitrogen. The section and surface was observed by SEM. Scan electron microscope: type Inspect.F, FEI Co., Holland.

\section{RESULTS AND DISCUSSION}

Crystalline orientation in high magnetic field: The polyacrylonitrile precursor contains cyano-group side group whose polarity was strong. Due to the strong repulsive force generated by cyano-group, the main carbon chain formed helical round rod whose diameter and length were about $0.6 \mathrm{~nm}$ and 10$100 \mathrm{~nm}$, respectively. Based on the attraction among macromolecular chains, several or dozen of round rod aligned tightly and forms crystal. It was reported that the polyacrylonitrile precursor was hexagonal cell. If the crystalline orientation was too low, i.e. the crystal align along different directions, the voids would generate among crystals.

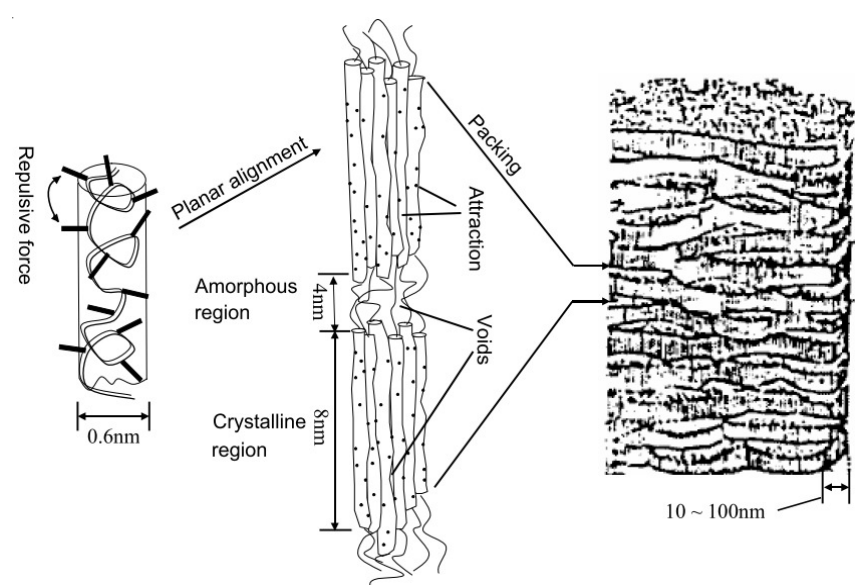

Fig. 1. Schematic diagram of the structure of polyacrylonitrile precursor

In the study, the polyacrylonitrile precursor was treated in $0 \mathrm{~T}, 8 \mathrm{~T}, 12 \mathrm{~T}, 16 \mathrm{~T}$ high magnetic field for $10 \mathrm{~min}, 30 \mathrm{~min}$, 50 min respectively, the crystalline orientation was measured by XRD azimuth angle scan and the result was showed in Fig. 2. It could be seen in the figure that the crystalline orientation increased as the magnetic field intensity enhanced when the processing time were the same. However, the orientation increased as the processing time extended, but the growth range reduced gradually, which could be deduced by the slope of 


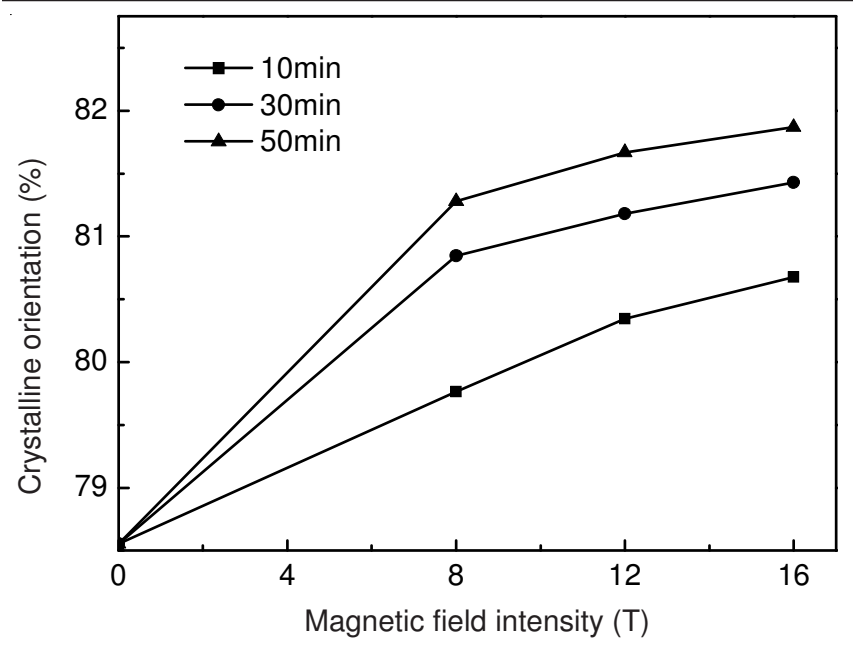

Fig. 2 . Crystalline orientation of polyacrylonitrile precursors processed in $0 \mathrm{~T}, 8 \mathrm{~T}, 12 \mathrm{~T}, 16 \mathrm{~T}$ magnetic field for $10 \mathrm{~min}, 30 \mathrm{~min}, 50 \mathrm{~min}$

curves. The result could be understood from the view of packing structure and steric hindrance. At the beginning, the alignment of molecular chain was relatively incompact and there was adequate free space for the movement of segment. So the growth rate of orientation was relatively fast in the initial stage. As the orientation increased, the alignment of segment became to be hard-packed and the free space decreased. The closest packing of segment generated steric hindrance effect which made the rotation of crystalline difficult. Moreover, most of the movement of segment had completed in the initial stage and they had no response to the magnetic field any more once they rotated into position. In the later stage, the movement of segment was slight and the orientation did not change any more.

The reasons for crystalline orientation and the changes of crystalline orientation could be interpreted through the three theories below.

Magnetostatic energy: The cyano-group side group which contained triple bond had two $p$ orbitals who didn't participate in hybridization in $\mathrm{C}$ and $\mathrm{N}$ atom, respectively. The four non-hybrid $p$ orbitals formed two $\pi$ bonds between which the electrons could move freely and form quasi-ring current induced by high magnetic field. The electron ring current was accelerated and produced a magnetic field whose direction was opposite to the external magnetic field in high magnetic field that was regarded as the symbol of diamagnetism. So a cyano-group could be regarded as a magnetic dipole. Because there was an angle between the magnetic dipole and external magnetic field, the magnetic dipole suffered torque force coming from external magnetic field unless the angle became zero. That was to say, the cyano-group possessed magnetostatic energy which could be calculated as

$$
\mathrm{F}=-\mu_{0} \mathrm{MH} \cos \theta
$$

In the formula, $\mathrm{F}$ was the intensity of magnetostatic energy, $\mu_{0}$ was permeability of vacuum, $M$ was magnetization intensity, $\mathrm{H}$ was external magnetic field intensity and $\theta$ was the angle between the magnetic dipole and external magnetic field. When $\theta=0^{\circ}, \mathrm{F}=-\mu_{0} \mathrm{MH}$ was the minimum and when $\theta$ $=180^{\circ}, \mathrm{F}=+\mu_{0} \mathrm{MH}$ was the maximum. The result showed the intensity of magnetostatic energy decreased as $\theta$ reduced that implied the system tended to be stable. Therefore, all the cyanogroups were induced to align along the uniform direction and the orientation increased.

Boltzmann formula: It had reported that in high magnetic field the degree of orientation was determined by the Boltzmann factor and the formula was

$$
\mathrm{f}_{\mathrm{c}}=\exp \left(-\Delta \chi_{\mathrm{m}} \mathrm{H}^{2} / 2 \mathrm{RT}\right)
$$

where $\Delta \chi_{\mathrm{m}}$ was the magnetic conductivity and $\mathrm{H}$ was the magnetic field intensity ${ }^{12}$. It was obvious to detect that as to the same sample both $\Delta \chi_{\mathrm{m}}$ and $\mathrm{T}$ were the same respectively, $\mathrm{f}_{\mathrm{c}}$ was determined by the magnetic field intensity $\mathrm{H}$. In the study, the result that the crystalline orientation increased as the magnetic field intensity rose at different processing time accorded with the theory definitely. Moreover, $\mathrm{f}_{\mathrm{c}}$ was in direct ratio with $-\Delta \chi_{\mathrm{m}} \mathrm{H}^{2} / 2 \mathrm{RT}$ that meant the variable $-\Delta \chi_{\mathrm{m}} \mathrm{H}^{2}$ was positive value. Because $H$ was positive, so the magnetic conductivity $\Delta \chi_{\mathrm{m}}$ was negative. Based on the result, it could be concluded that polyacrylonitrile precursor was diamagnetic anisotropy. And the result happened to coincide with the model in Fig. 3 completely.

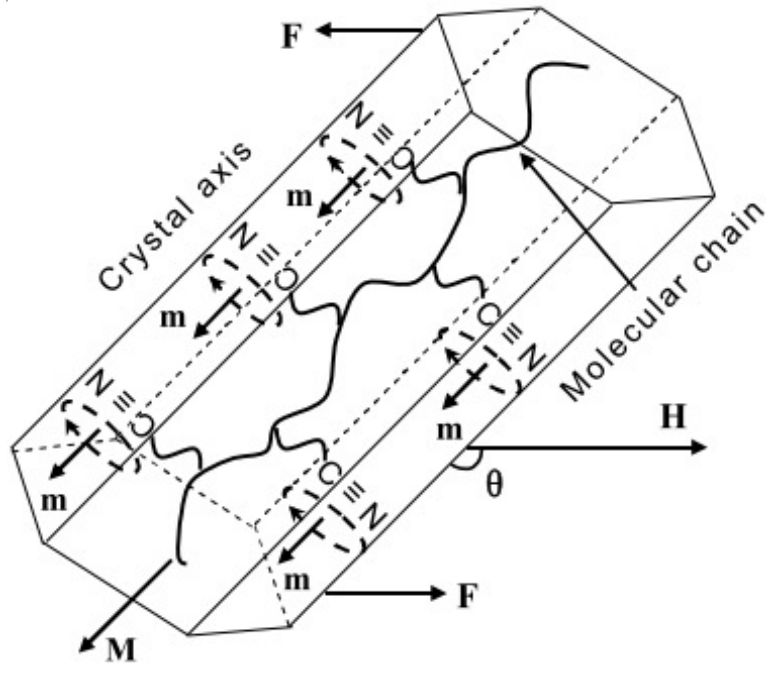

Fig. 3. Schematic diagram of magnetostatic energy of polyacrylonitrile crystal

Moment of force: From the moment of force point of view, the result could also be explained reasonably. Based on the analysis in magnetostatic energy, the polyacrylonitrile crystal unit could be regarded as a magnetic dipole and it was given a force in the magnetic field and the moment of force $\mathrm{L}$ could be calculated as

$$
\mathrm{L}=\frac{\mathrm{m}}{\mathrm{H} \cos \theta}
$$

where $\mathrm{m}$ was magnetic pole strength, 1 was the length of dipole. $\mathrm{L}$ was directly proportional to $\mathrm{H}$ when the other variables were the same. Higher L implied more orientation and the result happened to accord with the conclusion at which arrived through Boltzmann factor.

Overall orientation in high magnetic field: The polyacrylonitrile precursor was not a complete crystal and it contained quite a number of amorphous region. Generally the degree of crytallinity was $50-60 \%$ or so. Due to the chemical composition in crytallinity and amorphous region were the 
same, the magnetic field should had effect on the amorphous region, too. The overall orientation of samples processed in different magnetic fields was measured by infrared dichroism.

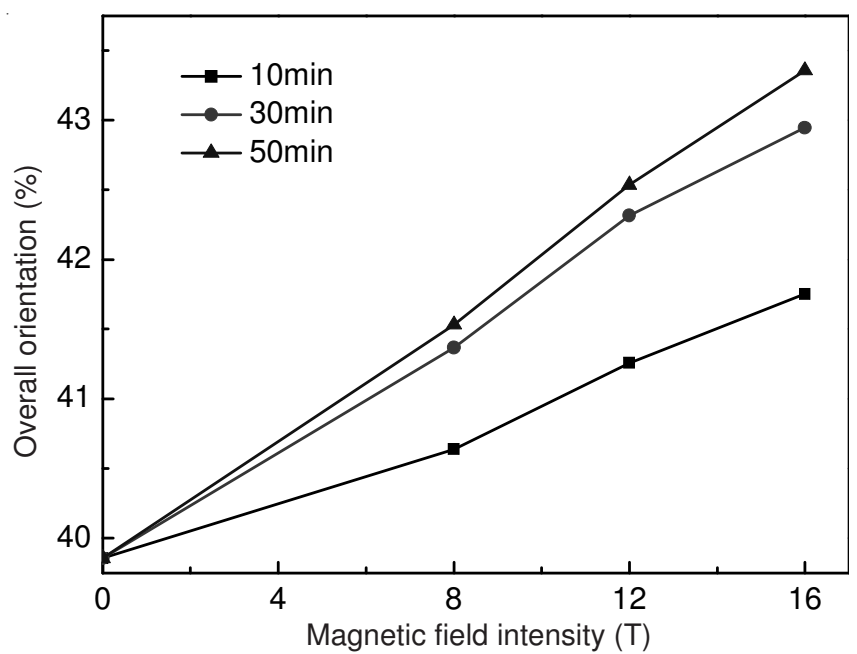

Fig. 4. Overall orientation of polyacrylonitrile precursors processed in $0 \mathrm{~T}$, $8 \mathrm{~T}, 12 \mathrm{~T}, 16 \mathrm{~T}$ magnetic field for $10 \mathrm{~min}, 30 \mathrm{~min}, 50 \mathrm{~min}$

When the processing time was the same, the overall orientation increased as the magnetic field intensity enhanced. The result was in accord with the former analysis about the relationship between the orientation and the intensity of external magnetic field. It testified the conclusion above that the stronger magnetic field brought about more orientation further.

The rate of growth was used to characterize the change range of orientation which was calculated as

$$
R=\left(d_{p}-d_{u}\right) / d_{u}
$$

where $\mathrm{R}$ was the rate of growth, $\mathrm{d}_{\mathrm{u}}$ was the degree of crystalline or overall orientation of unprocessed sample, $\mathrm{d}_{\mathrm{p}}$ was the degree of crystalline or overall orientation of processed sample.

Compared with the crystalline orientation, the rate of growth of the overall orientation was bigger in the same processing condition. According to the results above, when the processing time was $30 \mathrm{~min}$ in $8 \mathrm{~T}$ magnetic field, the rate of growth of crystalline orientation was $2.92 \%$ and that of overall orientation was $3.79 \%$. When the time extended to 50 min, the values were 3.47 and $4.21 \%$. The result implied that the amorphous region tended to align packing and contributed to the orientation. It was an assertive evidence to the result that amorphous region could response to the magnetic field. It could also be shown in Fig. 5 that the amorphous region was more susceptible to the change of magnetic field. Theoretically, it had been verified that the packing of amorphous region was more incompact than the crystallinity region. The loose structure meant less repulsive force and more free space among the molecular chains. So the orientation of amorphous region was easier compared to crystallinity region. Realistically, it was obvious to see that as the magnetic field intensity enhanced, the slope of overall orientation rate of growth was more than that of crystalline orientation which meant overall orientation had an bigger increasing tendency. Base on the analysis, it could be inferred that the increase of overall orientation resulted from amorphous region mainly.

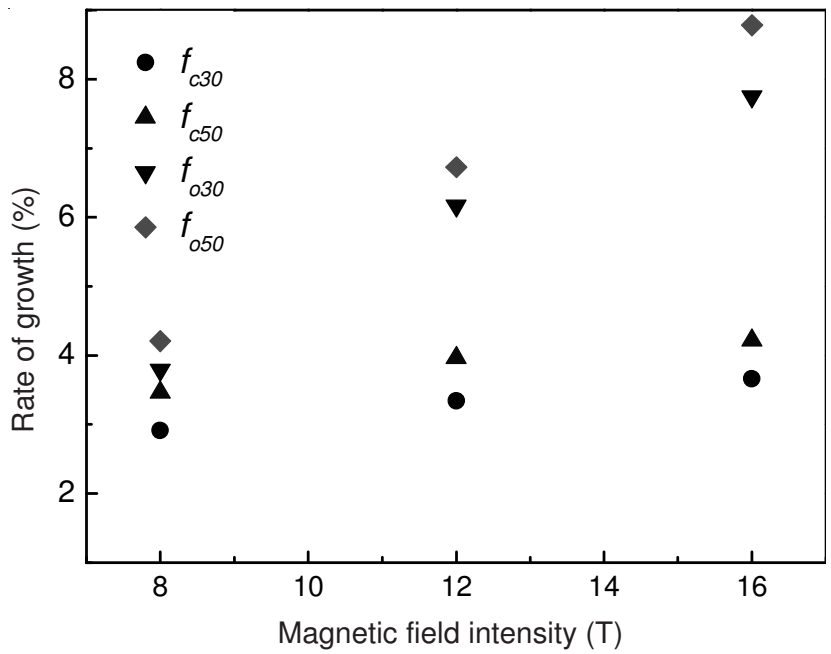

Fig. 5. Rate of growth of orientation in $8 \mathrm{~T}, 12 \mathrm{~T}, 16 \mathrm{~T}$ magnetic field for $30 \mathrm{~min}, 50 \mathrm{~min}: \mathrm{f}_{\mathrm{c} 30}$ - crystalline orientation of samples processing for $30 \mathrm{~min}, \mathrm{f}_{\mathrm{c} 50}$ - crystalline orientation of sample processing for 50 min, $\mathrm{f}_{030}$ - overall orientation of sample processing for $30 \mathrm{~min}, \mathrm{f}_{050}$ - overall orientation of sample processing for $50 \mathrm{~min}$

Other properties in high magnetic field: The high magnetic field had the effect not only on the crystalline and overall orientation, but on other properties of the polyacrylonitrile precursor. Through the density, DSC and SEM measures, the changes could be detected obviously.

Density: The densities of polyacrylonitrile precursor processed in $0 \mathrm{~T}, 8 \mathrm{~T}, 12 \mathrm{~T}, 16 \mathrm{~T}$ magnetic field in $130^{\circ} \mathrm{C}$ were measured by density gradient method. The results was showed in Fig. 6.

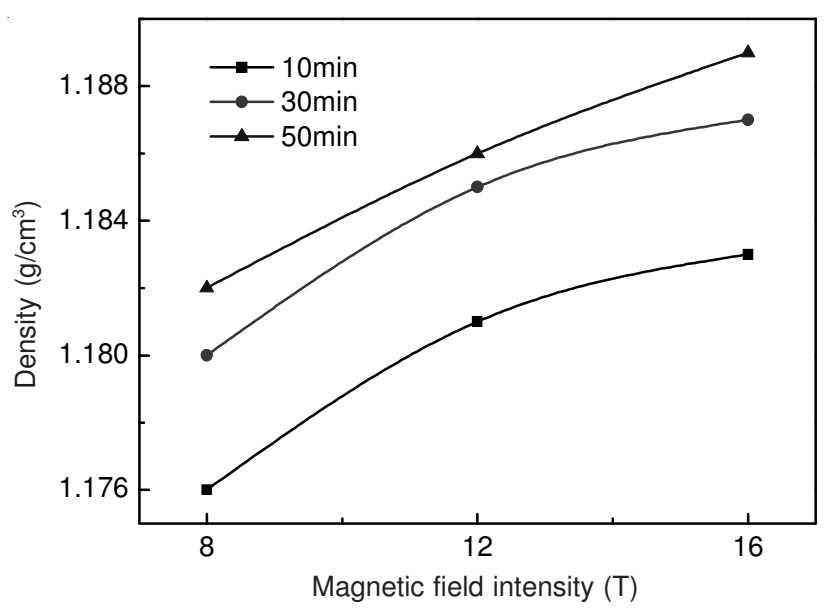

Fig. 6. Density of samples processed in $8 \mathrm{~T}, 12 \mathrm{~T}, 16 \mathrm{~T}$ magnetic field for $10 \mathrm{~min}, 30 \mathrm{~min}, 50 \mathrm{~min}$

In the figure, the density increased as the magnetic field intensity enhanced. There were some voids inside the polyacrylonitrile precursor due to the incompact packing of crystalline and the random arrangement of amorphous region, which caused the density decrease. After processed by high magnetic field, the voids decreased or disappeared because the crystalline tended to close-packing as the orientation modified. The theory could be the explanation for the increase of density. In addition, the increment speed of density accorded with the modification of orientation, which verified the relationship between density and orientation further. 
DSC: The thermal behaviour of different samples were measured by DSC at the rate of $10{ }^{\circ} \mathrm{C} / \mathrm{min}$ (Fig. 7). In the spectrum, it was obvious to see that the exothermic peak shifted to high temperature gradually as the magnetic field intensity increased. The exothermic peak resulted from the cyclization reaction which was testified to happen in amorphous region firstly due to the incompact structure. When the packing of molecular chain tended to be tight, it became hard to initiate the reaction that caused the peak temperature high. So the shift of exothermic peak implied the magnetic field process improved the compactness of polyacrylonitrile molecular packing. And the close packing had some relation with the increase of orientation.

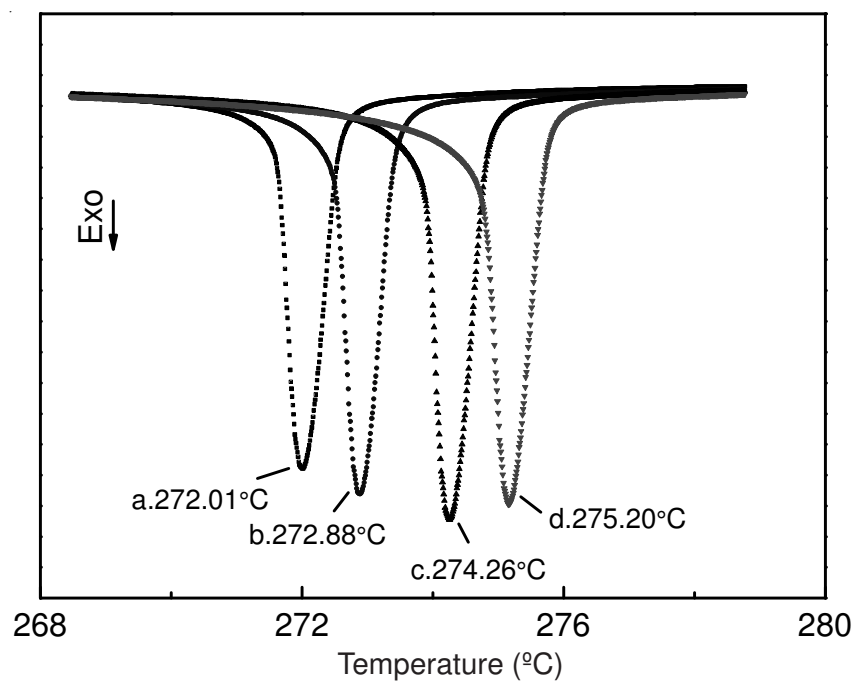

Fig. 7. DSC curves of samples processed in different time: (a) 0 min, (b) $10 \mathrm{~min}$, (c) $30 \mathrm{~min}$, (d) $50 \mathrm{~min}$

SEM: The fracture and surface of processed and original precursors were observed by SEM respectively (Fig. 8). It was obvious to detect that in the fracture micrographs the voids were small and evenly distributed in processed sample, while the voids were relatively big and unevenly distributed in original one. It resulted from the realignment of molecular chain and was an evidence to prove that the molecular chain oriented in high magnetic field. In the surface micrographs, the processed sample showed a more roughish surface. The phenomenon may come from the movement of molecular chain to fill the interspace in high magnetic field. As the arrangement tended to be tight, some interspace was filled and some grooves appeared in the surface, which caused the surface rough.

\section{Conclusion}

In the study, the polyacrylonitrile precursor for carbon fiber was processed in different steady-state high magnetic fields in recrystallization. Through the analysis of crystalline and overall orientation, it was affirmative to infer that polyacrylonitrile precursor could be induced oriented. And the degrees of both crystalline and overall orientation increased as the magnetic field intensity enhanced. The mechanism of crystalline orientation accorded with the magnetostatic energy, Boltzmann factor, moment of force theories definitely. In addition, the Boltzmann factor theory testified the magnetic conductivity $\Delta \chi_{\mathrm{m}}$ of polyacrylonitrile precursor was negative which meant polyacrylonitrile precursor was diamagnetic
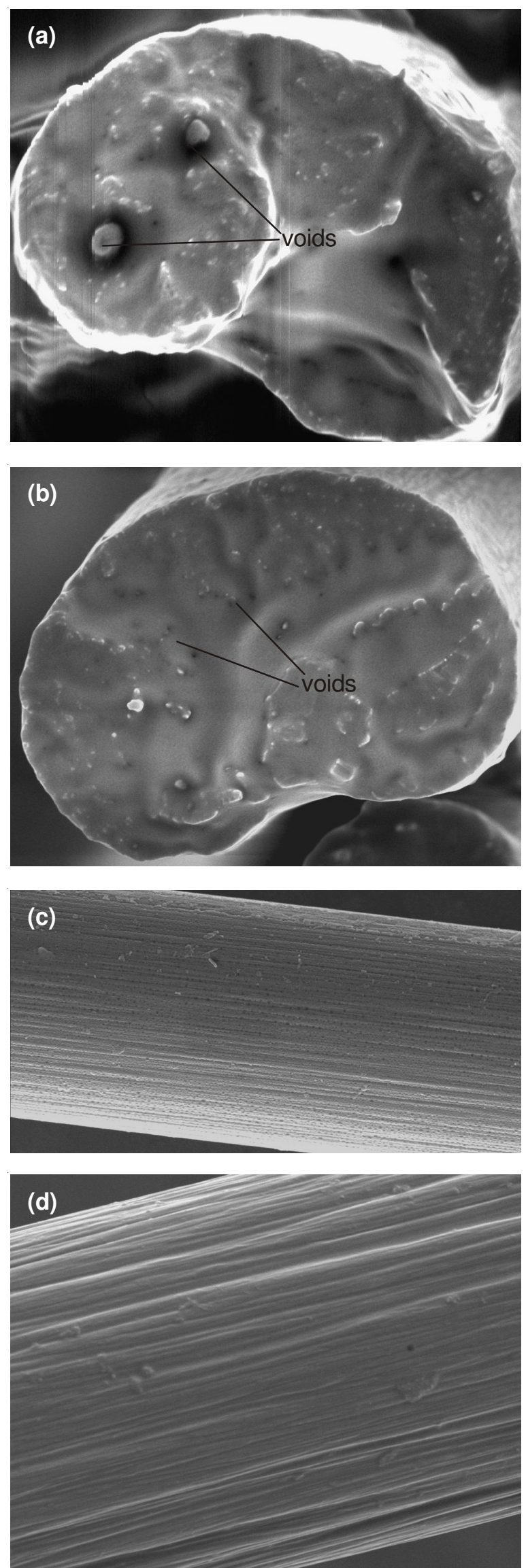

Fig. 8. SEM micrographs of fracture and surface of processed and original precursors: (a) fracture of original precursor, (b) fracture of processed precursor, (c) surface of original precursor, (d) surface of processed precursor 
anisotropy and the result coincided with the magnetostatic energy theory. The rate of growth of overall orientation was bigger than that of crystalline orientation and the result testified the amorphous region was more susceptible to magnetic field and the increase of overall orientation resulted from amorphous region mainly. Due to the change of orientation, correlative other properties modified also. As the magnetic field intensity enhanced, the density increased, the exothermic peak shifted to high temperature, the voids of surface and fracture disappeared. All the changes testified the effect of steady-state high magnetic field on polyacrylonitrile precursor further.

\section{REFERENCES}

1. W.X. Zhang, J. Liu and G. Wu, Carbon, 41, 2805 (2003).

2. Y.F. Wen, J.Q. Guo, Z.M. Gao, X.S. Li, X. Cao and Y.G. Yang, New Carbon Mater., 24, 153 (2009).
3. L.Z. Zhang, C.X. Lv, Y.G. Lv, G.P. Wu and F. He, New Carbon Mater, 20, 144 (2005).

4. J. Liu, J. Li, L. Wang, Z.K. Ma and J.Y. Liang, New Carbon Mater, 23, 177 (2008).

5. H.P. Xu, Y.P. Sun and X.M. Chen, New Carbon Mater, 20, 312 (2005).

6. H. Rennhofer, D. Loidl, S. Puchegger and H. Peterlik, Carbon, 48, 964 (2010).

7. T. Kimura, T. Kawai and Y. Sakamoto, Polymer, 41, 809 (2000).

8. J. Torbet, Y.F. Nicolau and D. Djurado, Synth. Met., 101, 825 (1999).

9. H. Yonemura, K. Yuno, Y. Yamamoto, S. Yamada, Y. Fujiwara and Y. Tanimoto, Synth. Met., 159, 955 (2009).

10. T. Kawai, R. Iijima, Y. Yamamoto and T. Kimura, Polymer, 43, 7301 (2002).

11. H.P. Schad, G. Baur and G. Meier, J. Chem. Phys., 71, 3174 (1979).

12. A.P. Chiriac and C.I. Simionescu, Prog. Polym. Sci., 25, 219 (2000). 